* Corresponding author E-mail address:milan.zmindak@fstroj.uniza.sk (Milan Žmindák, prof., MSc., PhD.)

Article information Article history: AMS-Volume15-No.3-00115-11 Received 28 March 2011 Accepted 24 May 2011

\section{Application of Fast Multipole Boundary Method in Elastostatic Problems}

\author{
Milan Žmindák*, Pavol Novák, Daniel Riecky \\ University of Žlina, Faculty of Mechanical Engineering, Department of Applied Mechanics, Univerzitná 1, 01026 Žllina
}

\section{BIOGRAPHICAL NOTES}

Milan Žmindák, prof. MSc., PhD., professor of Applied Mechanics, Deputy Head of Department of Applied Mechanics, Faculty of Mechanical Engineering, University of Žilina. He graduated from the Faculty of Mechanical Engineering CTU in Prague, field of study Applied Mechanics. In 1992 he was at the Institute of Materials and Machine Mechanics SAS in Bratislava awarded the scientific degree of candidate of technical sciences in the field of solid mechanics and rigid bodies and the environment. In 1995 he was at Faculty of Mechanical Engineering in Žilina habilited in the field of Applied Mechanics. In 2008 he was appointed President of the Slovak Republic Professor of Applied Mechanics. The main subject of his research activities are modeling of composite structures using finite element method, boundary element method and meshless methods. He is member of Central European Association for Computational Mechanics (CEACM), Slovak Society for Mechanics and Czech Society for Mechanics. He is the author and co-author of two monographs, four university textbooks, five chapters in books, and a few tens papers in refereed journals. Most cited work by $\mathrm{SCl}$ is paper Simulation of the aquaplane problem published in International Journal Computers and Structures.

Pavol Novák, MSc., PhD., (born 1972) Is a teacher at the Faculty of Mechanical Engineering, Department of Applied Mechanics, University of Žilina. In 1996 he graduated at the Faculty of Mechanical Engineering, specialization Applied Mechanics, in 2010 he obtained postgraduate academic degree philosophiae doctor (PhD.) in specialization Applied Mechanics. He is member of Central European Association for Computational Mechanics (CEACM) and Slovak Society for Mechanics. The author deals with problems in the field of continuum mechanics (fluids and solids) and he is focused on numerical modeling of welding processes. He is author and co-author of 5 scientific articles in refereed journals and other 20 scientific articles.

Daniel Riecky, MSc., (born 1984) Is internal PhD student at Faculty of Mechanical Engineering, Department of Applied Mechanics, University of Žilina. In 2009 he graduated at the Faculty of Mechanical Engineering, specialization Applied Mechanics. The main subject of his research activity is modeling of composite materials by meshless methods. He is coauthor of 10 scientific contributions in conference proceedings and journals.

\section{KEY WORDS}

Boundary Element Method, Fast Multipole Method, Reciprocity Based FEM.

\section{ABSTRACT}

The boundary element method (BEM) is a numerical method for solving boundaryvalue or initial-value problems formulated by use of boundary integral equations. In 
the BEM, only the boundaries - that is, surfaces for three-dimensional problems or curves for two dimensional (2D) problems - of a problem domain need to be discretized. However the boundary element method (BEM) has been limited to solving problems with a few thousand degrees of freedom (DOFs) on a personal computer. This is because the conventional BEM, in general, produces dense and nonsymmetric matrices. The main idea of the fast multipole (FM) BEM is to employ iterative solvers to solve the BEM system of equations. Using this method we can solve models with more than one million equations on a laptop computer. In this paper, the governing equations for elasticity problems are reviewed first. Numerical examples are provided to demonstrate the accuracy and efficiencies of fast multipole method (FMM) for solving 2D elasticity problems.

\section{Introduction}

The finite element method (FEM) is the most significant numerical procedures commonly applied to analyses continuum mechanics models $[1,2]$. The boundary element method (BEM) is now established in many engineering disciplines as an alternative numerical technique to FEM. The attraction of BEM can be largely attributed to the reduction in dimensionality of the problem. On the other hand, this basic BEM behavior brings a loss generality in comparison with the FEM. The loss of partial generality of the BEM is balanced by its high accuracy of results, especially for stress concentration problems. Namely, the solution at an internal point of analysed domain is exactly expressed though the boundary values and no discretization of domain is required. This is the main reason why the BEM is the most computational method for solution of crack problems [3]. For 2-D elasticity problems using fast multipole BEM, there are several approaches as well Greengard et al. [4] used a fast multipole formulation for directly solving the biharmonic equations in 2-D elasticity. They applied Sherman's complex variable formulation to solve the biharmonic equation and presented several interesting large-scale problems. Peirce and Napier [5] developed a spectral multipole approach, that shares some common features with

the FMMs. In their approach, a set of background grids are generated and Taylor series expansions of the kernels are used to compute the integrals at the grid points. Interpolations of these values give the values at the collocation points. The alternative stress and displacement models are the hybrid - Trefftz finite element formulations and reciprocity based FEM $[6,7]$. In recent years, more and more attention has been paid to researches on the meshless (or meshfree) method, which makes it a hot direction of computational mechanics $[8,9]$. The meshless method is t he approximation based on nodes; then the large deformation and crack growth problems can be simulated with the method without the re-meshing technique. The meshless method has some advantages over the traditional computational methods, such as FEM and BEM. In this paper, a new FMM BEM formulation is presented for 2-D elasticity problems based on the direct BIE formulation. The displacement and traction kernels are represented using the two complex analytic functions in 2-D elasticity [10]. The FMM was developed to increase the efficiency of the boundary type numerical models by reducing computations for the interaction of far fields. However, near field integrals still have to be solved by classical BEM and the boundaries are also discretized by elements. The FMM improves considerably numerical models based on the BEM especially for far field interaction. However, the near field interaction is solved by classical procedure and the models for composites reinforced by many interacting fibres and particles require still very efficient computers.

\section{Conventional Boundary Element Method}

The basis of any boundary integral equation (BIE) formulation is rooted in classical elasticity theory as the reciprocal work theorem of Betti (1872) and the application of elastic potentials to satisfy equilibrium by Somigliana (1885). Much of the literature in the past twenty years of BIE formulations has made use of the method of weighted residuals. Although the BEM has enjoyed the reputation of easy meshing in modelling many problems with complicated geometries, its efficiency in solutions has been a serious problem for analyzing large-scale models.

The current discussion will treat isotropic elastic continua (Fig.1). Equilibrium in the presence of body forces (per unit volume) is given by the gradient of the stress tensor $\sigma_{\mathrm{ij}}$ as:

$$
\frac{\partial \sigma_{i j}}{\partial x_{j}}+X_{i}=0
$$




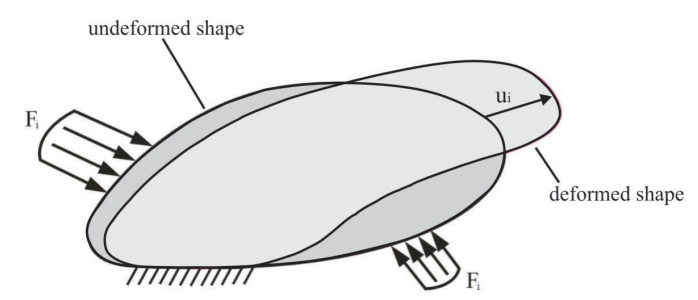

Fig. 1: Deformed solid.

The strain tensor is defined as

$\varepsilon_{k l}=\frac{1}{2}\left(u_{k, l}+u_{l, k}\right)$

Taking Hook's law for the material we obtain stress -strain relation

$\sigma_{i j}=C_{i j k l} \varepsilon_{k l}$

where tensor of material constants for an isotropic body is given as [3]

$C_{i j k l}=\lambda \delta_{i j} \delta_{k l}+\mu\left(\delta_{i k} \delta_{j l}+\delta_{i l} \delta_{j k}\right)$

Substituting eqs. (3) and (2) into (1) we obtain the Navier equation of equilibrium in terms of the displacements

$C_{i j k l} u_{k, l}+X_{i}=0$

in a general form or in the form valid only for an isotropic material

$\mu u_{i, k k}+(\lambda+\mu) u_{k, k i}+X_{i}=0$

The body surface can be split into two non-overlaping parts depending on the boundary conditions. Denote the part where displacements are prescribed by $\Gamma_{u}$ and part where tractions are described $\boldsymbol{\Gamma}_{t}$ (Fig. 2), then $\mathrm{BC}$ are given by following expressions:

\section{- Displacements $B C$}

$u_{i}(y)=\bar{u}_{i}(y) \quad y \in \partial \Gamma_{u}$

- Tractions $B C$

$T_{i}^{n}(y)=\bar{t}_{i}(y)=\sigma_{i j}(y) n_{j}(y) \quad y \in \partial \Gamma_{t}$

\section{- Displacements and tractions BC}

$u_{i}(y)=\bar{u}_{i}(y) \quad y \in \partial \Gamma_{u}$

$T_{i}^{n}(y)=\bar{t}_{i}(y)=\sigma_{i j}(y) n_{j}(y) \quad y \in \partial \Gamma_{t}$

On Fig.3 is described elasticity problem. Equa- tions (1) (2), (3) represents elasticity equations. The equations (4) and (5) are boundary conditions.

Fundamental solutions are singular solutions to eq. (6) for the infinite region if body force is represented by the Dirac delta function.

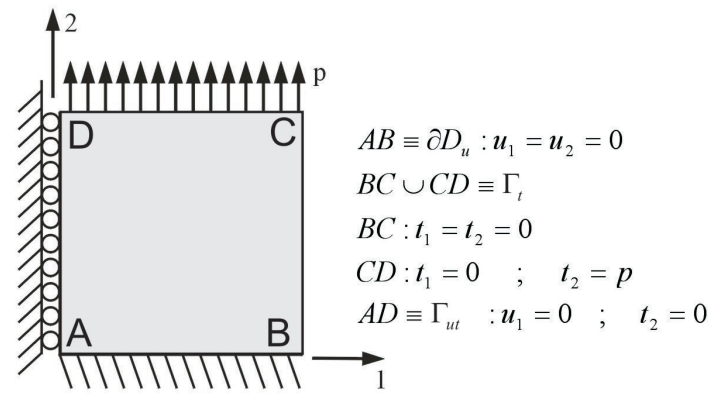

Fig. 2: Boundary conditions.

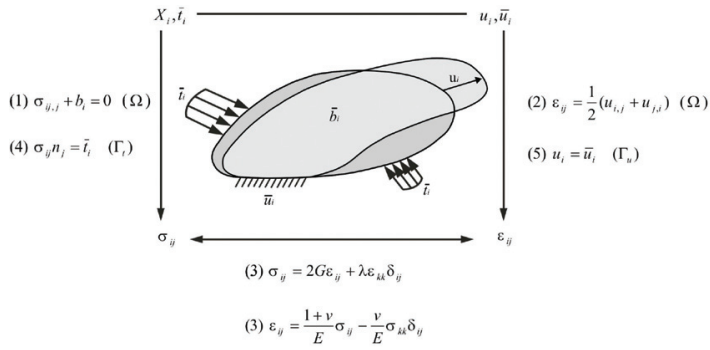

Fig. 3: Elastic problem.

Introducing the Poisson number $u$, the Lame constant $\lambda(\lambda=2 \mu \nu /(1-2 \nu))$ can be eliminated, and the Kelvin solution becomes [3]

$U_{i j}(\mathbf{x}, \mathbf{y})=\frac{1}{16 \pi \mu(1-\nu) r}$.

$\left[(3-4 \nu) \delta_{i j}+r_{, i} r_{j}\right]$

The traction vector is defined as a scalar product of stresses $\sigma_{\mathrm{ij}}$ and normal vector $n_{\mathrm{j}}$

$t_{i}(x)=\sigma_{i j}(x) n_{j}(x)=c_{i j k l} n_{j}(x) \varepsilon_{k l}(x)$

Then, the traction vector corresponding to fundamental displacements (10) has the form

$$
\begin{aligned}
& T_{i j}=\mu\left(U_{i j, k}+U_{k j, i}\right) n_{k}+\lambda n_{i} U_{k j, k}= \\
& =\frac{1-2 \nu}{8 \pi(1-\nu) r^{2}} . \\
& {\left[r_{j} n_{i}-r_{, i} n_{j}-\left(\delta_{i j}+\frac{3}{1-2 \nu} r_{i} r_{j}\right) n_{k} r_{, k}\right]}
\end{aligned}
$$

In the following we shall be concerned with twodimensional problems of elastostatics. The equilib- 
rium equation in $2-d$ problem is similar to that for 3-d given by eq. (5). Constitutive equation can be written as

$\sigma_{i j}=2 \mu \varepsilon_{i j}+\alpha \delta_{i j} \varepsilon_{k k}$

where the constant being defined as

$\alpha= \begin{cases}\lambda & \text { for plane strain } \\ \frac{2 \mu \lambda}{\lambda+2 \mu} & \text { for plane stress } .\end{cases}$

\section{Discretization of the Bie}

As it is generally not possible to compute the boundary integrals in the integral representations as well as to solve the boundary integral equations analytically, a numerical approach is adopted.

Recent advances, such as the use of isoparametric boundary elements, superior numerical integration schemes and the careful analytical treatment of singularity cases, have had a major impact on the competitiveness of the BEM in routine linear, or non-linear two- and three-dimensional analyses.

The numerical solution of a problem follows the usual procedures of the BEM. The boundary is discretized into elements using piecewise polynomial approximations of the boundary geometry, displacements and tractions (Fig. 4). The simplest interpolation function is the constant approximation, where boundary displacements and tractions are constant over each element. In general, howev$\mathrm{er}, u_{\mathrm{i}}$ and $t_{\mathrm{i}}$ can have a linear or higher-order variation. In addition, their polynomial behaviour need not be of the same order and, for instance, it may be more consistent to take $t$. of one order less than $u_{\mathrm{i}^{\prime}}$ since a traction is given by the derivative of a displacement. In practice it is simpler to take both functions $u_{\mathrm{i}}$ and $t_{\mathrm{i}}$ of the same order.

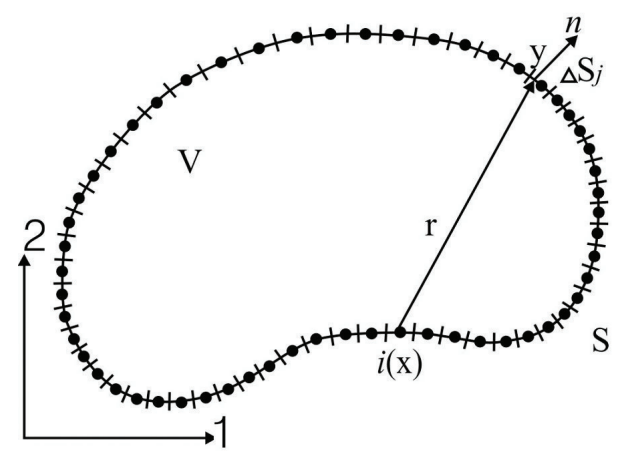

Fig. 4: Discretization of the boundary S using constant element.

\section{Fast Multipole Boundary Element Method}

For derivation FM BEM we use direct conventional boundary integral equation (CBIE) for 2-D elastostatic problems [11]

$C_{i j}(\mathbf{x}) u_{j}(\mathbf{x})=\int_{S}\left[U_{i j}(\mathbf{x}, \mathbf{y}) t_{j}(\mathbf{y})-\right.$

$\left.-T_{i j}(\mathbf{x}, \mathbf{y}) u_{j}(\mathbf{y})\right] d s(\mathbf{y}) \quad \forall \mathrm{x} \in S$

where $u_{\mathrm{i}}$ and $t_{\mathrm{i}}$ are the displacement and traction, respectively; $S$ the boundary of domain $V$ (Fig. 5); $C_{i j}(\mathbf{x})$ coefficients that are equal to if $S$ is smooth around $\mathbf{x}\left(\delta_{i j}\right.$ is the Kronecker $\left.\delta\right)$; and $i, j=1,2$ in 2-D cases. The kernel functions $U_{i j}(\mathbf{x}, \mathbf{y})$ and $T_{i j}(\mathbf{x}, \mathbf{y})$ are given by equations (10) and (12).

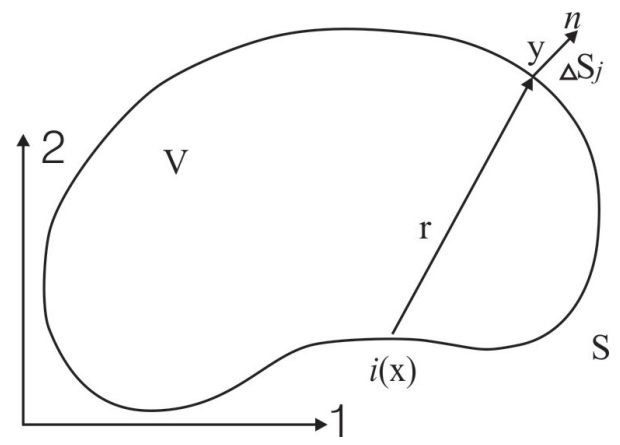

Fig. 5: Domain $V$ and boundary $S$.

Two integrals in CBIE (14) can be represented in complex variables readily if we write the fundamental solution $U_{i j}(\mathbf{x}, \mathbf{y})$ and $T_{i j}(\mathbf{x}, \mathbf{y})$ in complex notation by using results in $2 \mathrm{D}$ elasticity.

Then complex variables, the displacement field $U=U_{1}+i U_{2}$ at a field point $\mathrm{z}$ because of a point of force $P=P_{1}+i P_{2}$ can be written as force at source point $z_{0}$ can be written as

$$
\begin{aligned}
& U_{1}(z)+i U_{2}(z)=\frac{1}{4 \pi \mu(1+\kappa)}\left\{-\kappa P\left[\log \left(z_{0}-z\right)+\right.\right. \\
& \left.\left.+\overline{\log \left(z_{0}-z\right)}\right]+\frac{\bar{P}\left(z_{0}-z\right)}{\overline{z_{0}-z}}\right\}
\end{aligned}
$$

We can show that the first integral in CBEI can be written in following complex form by applying eq. (15) (with no body force)

$$
\frac{1}{2} u\left(z_{0}\right)=D t\left(z_{0}\right)-D u\left(z_{0}\right)
$$

where $u=u_{1}+i u_{2}$ is the complex representation of the displacement field and boundary $S$ is assumed 
to be smooth at source point $z_{0}$. In preceding equation:

$$
\begin{aligned}
& D_{t}\left(z_{0}\right)=\left[\int_{S} U_{1 j}(\mathbf{x}, \mathbf{y}) t_{j}(\mathbf{y}) d S(\mathbf{y})\right]+ \\
& +i\left[\int_{S} U_{2 j}(\mathbf{x}, \mathbf{y}) t_{j}(\mathbf{y}) d S(\mathbf{y})\right]
\end{aligned}
$$

representing the first integral with the $U$ kernel in $\mathrm{CBIE}$, and

$$
\begin{aligned}
& D_{u}\left(z_{0}\right) \equiv\left[\int_{S} T_{1 j}(\mathbf{x}, \mathbf{y}) u_{j}(\mathbf{y}) d S(\mathbf{y})\right]+ \\
& +i\left[\int_{S} T_{2 j}(\mathbf{x}, \mathbf{y}) u_{j}(\mathbf{y}) d S(\mathbf{y})\right]= \\
& =\frac{1}{1+\kappa} \int_{S}\left\{\kappa G^{\prime}\left(z_{0}, z\right) n(z) u(z)-\right. \\
& -\left(z_{0}-z\right) \overline{G^{\prime \prime}}\left(z_{0}-z\right) \overline{n(z)} \overline{u(z)}+ \\
& \left.+\overline{G^{\prime}\left(z_{0}, z\right)}[n(z) \overline{u(z)}+u(z)]\right\} d S(z)
\end{aligned}
$$

and representing the second integral with the $T$ kernel in CBIE, where $t=t_{1}+i t_{2}$ and $n=n_{1}+i n_{2}$ are complex traction and normal, respectively

$G\left(z_{0}, z\right)=-\frac{1}{2 \pi} \log \left(z_{0}-z\right)$

is the Green's function (in complex form) for 2D potential problems [11]. To derive the complex form hypersingular BIE (HBIE), we first note that the real variable traction $t_{i}$ on boundary $S$ is given by

$t_{i}=\sigma_{i j} n_{j}=\left[\lambda \delta_{i j} u_{k, k}+\mu\left(u_{i, j}+u_{j, i}\right)\right] n_{j}$

in which $\sigma_{i j}$ is the stress tensor and $\lambda=\frac{2 \mu \nu}{1-2 \mu}$ for plane/strain problems. It is interesting to note that this relation can be written in complex form as follows

$$
t(z)=2 \mu\left[\frac{1}{\kappa-1}\left(\frac{\partial u}{\partial z}+\frac{\partial \bar{u}}{\partial \bar{z}}\right) n+\frac{\partial u}{\partial \bar{z}} \bar{n}\right]
$$

in which $t, u$ and $n$ are the complex traction, displacement, and normal on boundary, $S$, respectively.

Applying (21), can be show HBIE can be written in the following complex form (with no body force)

$$
\frac{1}{2} t\left(z_{0}\right)=F t\left(z_{0}\right)-F u\left(z_{0}\right)
$$

$F_{t}\left(z_{0}\right)=2 \mu\left[\frac{1}{\kappa-1}\left(\frac{\partial D_{t}\left(z_{0}\right)}{\partial z_{0}}+\frac{\partial \overline{D_{t}\left(z_{0}\right)}}{\partial \bar{z}_{0}}\right) n\left(z_{0}\right)+\right.$ $\left.+\frac{\partial D_{t}\left(z_{0}\right)}{\partial \overline{z_{0}}} \overline{n\left(z_{0}\right)}\right]$

represents the first integral with the $K$ kernel in $\mathrm{HBIE}$, and

$$
\begin{aligned}
& F_{u}\left(z_{0}\right)=2 \mu\left[\frac{1}{\kappa-1}\left(\frac{\partial D_{u}\left(z_{0}\right)}{\partial z_{0}}+\frac{\partial \overline{D_{u}\left(z_{0}\right)}}{\partial \overline{z_{0}}}\right) n\left(z_{0}\right)+\right. \\
& \left.+\frac{\partial D_{u}\left(z_{0}\right)}{\partial \overline{z_{0}}} \overline{n\left(z_{0}\right)}\right]
\end{aligned}
$$

represents the second integral with the $H$ kernel in HBIE. Applying eqs. (17) and (18), we obtain the following explicit results

$F_{t}\left(z_{0}\right) \equiv\left[F_{1}(\mathbf{x})+i F_{2}(\mathbf{x})\right]_{t} \equiv$

$\equiv \int_{S} K_{1 j}(\mathbf{x}, \mathbf{y}) t_{j}(\mathbf{y}) d S(\mathbf{y})+$

$+i \int_{S} K_{2 j}(\mathbf{x}, \mathbf{y}) t_{j}(\mathbf{y}) d S(\mathbf{y})=$

$=\frac{1}{1+\kappa} \int_{S}\left\{\left[G^{\prime}\left(z_{0}, z\right) t(z)+\overline{G^{\prime}\left(z_{0}, z\right) t(z)}\right] n\left(z_{0}\right)+\right.$

$+\left[\kappa \overline{G^{\prime}\left(z_{0}, z\right)} t(z)-\right.$

$\left.\left.-\left(z_{0}-z\right) \overline{G^{\prime \prime}\left(z_{0}, z\right) t(z)}\right] \overline{n\left(z_{0}\right)}\right\} d S(z)$

$F_{u}\left(z_{0}\right) \equiv\left[F_{1}(\mathbf{x})+i F_{2}(\mathrm{x})\right]_{u} \equiv$

$\equiv \int_{S} H_{1 j}(\mathbf{x}, \mathbf{y}) t_{j}(\mathbf{y}) d S(\mathbf{y})+$

$+i \int_{S} H_{2 j}(\mathbf{x}, \mathbf{y}) t_{j}(\mathbf{y}) d S(\mathbf{y})=$

$=-\frac{2 \mu}{1+\kappa} \int_{S}\left(\left[G^{\prime \prime}\left(z_{0}, z\right) n(z) u(z)+\overline{G^{\prime \prime}\left(z_{0}, z\right) n(z) u(z)}\right] n\left(z_{0}\right)\right.$

$+\left\{\overline{G^{\prime \prime}\left(z_{0}, z\right)}[n(z) \overline{u(z)}+\overline{n(z)} u(z)]-\right.$

$\left.\left.-\left(z_{0}-z\right) \overline{G^{\prime \prime}\left(z_{0}, z\right) n(z) u(z)}\right\} \overline{n\left(z_{0}\right)}\right) d S(z)$

To show that complex variable CBIE is equivalent to real variable CBIE and complex variable HBIE is equivalent to real variable $\mathrm{HBIE}$, we can simply introduce the polar system $(r, \theta)$ with origin at $z_{0^{\prime}}$ notice that

$z-z_{0}=r e^{i \theta}, G^{\prime}=\frac{1}{2 \pi\left(z-z_{0}\right)}=\frac{e^{-i \theta}}{2 \pi r}$

and extract the real and imaginary parts of the results in complex variable BIEs.

The multipole expansions, local expansions and their translations related to Equation (17) in the new FM BEM are presented in [10].

where 


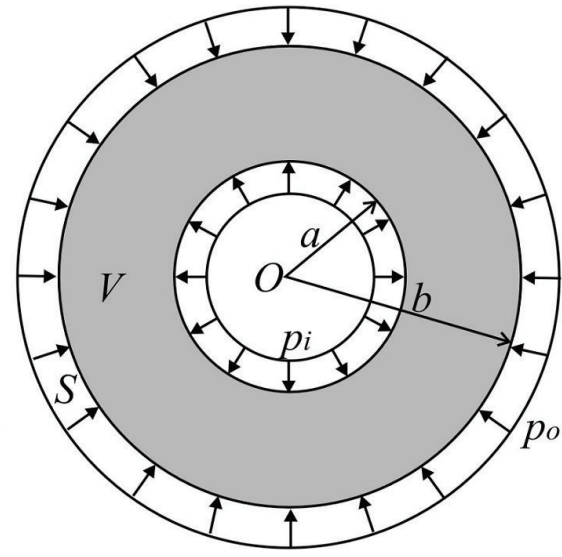

Fig. 6: A thick cylinder with pressure.

\section{Numerical Examples}

Several numerical examples are given in this section to demonstrate the accuracy and efficiency of the FM method for solving 2D elasticity problems. A first consider a thick cylinder under pressure loads (in the plane-strain case) as shown in Fig. 6. The inner pressure pi and outer pressure is $p_{0}$. In the case studied, $\mathrm{b}=2 \mathrm{a}, p_{i}=p_{0}=p$ and Poisson's ration $\nu=0.3$. We discretize the inner and outer boundaries with the same number of elements and run both the FM BEM code and conventional BEM code that also uses constant elements and analytical integrations. The conventional BEM code uses both the direct solver (LAPACK) and the iterative solver (GMRES) for solving the linear system. For the FM BEM, the numbers of terms for both multipole and local expansions were set to 20, the maximum number of elements in a leaf to 20 , and the tolerance for convergence of the solution to $10^{-6}$. All the FM BEM results converged in about 3 iterations without using any pre-conditioner in this example. The results of radial displacement component ur and hoop stress or at the inner boundary using both the FM BEM and the conventional BEM (with the direct solver) as the total number of elements increases from 200 to 4800 (degrees of freedom from 400 to 9600) are evaluated. As we can see, the results for both FM BEM and conventional BEM converge quickly to the exact solution [12] for the mesh with 360 constant elements with a relative error of less than $3 \%$. The results continue to improve with the increase in the number of elements. This example shows that the FM BEM is very efficient compared with the conventional BEM. In addition, the FM BEM results are equally accurate as the conventional BEM results, and they are very stable with the increase in the model size.

In second example, we further study of accuracy of the FM method using stress concentration problem - square with a circular hole at centre, as shown in Fig. 7. The edge length of the square plate is a and radius of the hole is $R=0.1 a$. The plate is loaded in the $x$-direction with a uniform load $\mathrm{p}$, and Poisson's ratio $=0.3$ in this study. The maximum (at point $A$ ) and minimum (at point $B$ ) hoop stresses on the edge of the hole are sought (Fig. 7) using both FM BEM code and ANSYS, a finite element method (FEM) package. In the BEM models, numbers of boundary elements on the edge of the hole increase while that on the outer edges of the plate is kept at 100, except for the last BEM model in which 200 elements are used on the outer edges of the plate. The numbers of terms for both multipole and local expansions

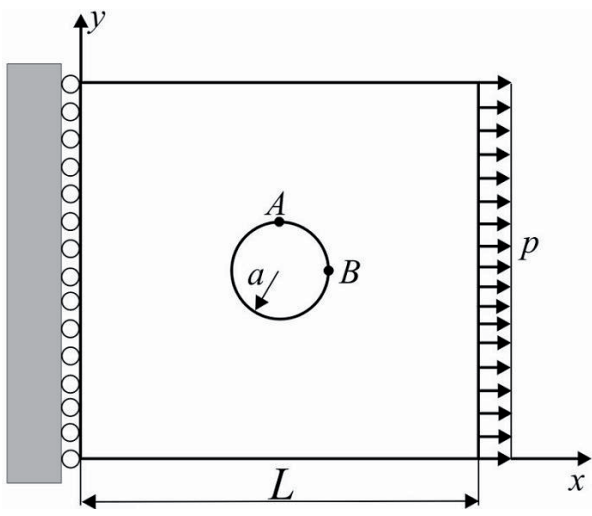

Fig. 7: A square plate.

were set to 20 , the maximum number of elements in a leaf to 100, and the tolerance for convergence to $10^{-6}$. All the FM BEM results converged in about 20 iterations. In the FEM models, 4-node quadrilateral elements are used in order to compare with the BEM models (which use constant boundary elements). In the FEM meshes, smaller elements are used near the hole while larger elements are used near the outer boundaries of the plate.

Tab. 1 shows the comparison of the computed hoop stresses at points $A$ and $B$. For an infinitely large plate with a hole, the hoop stress at point $A$ is $3 p$, while that at point $\mathrm{B}$ is $-p[12]$. The stress values for both FM BEM (with DOFs=1640) and FEM (with 
DOFs $=4522$ ) converged quickly to around $3.22 p$ at point $A$ and $-1.19 p$ at point $B$.

Further increases in the numbers of elements provided little improvements in the results. This example demonstrates again that the results using FM BEM code are accurate and stable. It should be pointed out that the element types used for both the BEM and FEM in this study are the simplest elements available. If higher-order elements such as quadratic elements are used, a few hundred elements should be sufficient for both the BEM and FEM to achieve the same accuracy as reported in this example. Comparison of the CPU times used for the two BEM approaches are plotted in Fig. 8, which shows the significant advantage of the FM BEM compared with conventional BEM with either a direct or an interactive solver. For example, for model with 9600 DOF, the FM BEM used only 3s of the CPU time, whereas the conventional BEM used $1480 \mathrm{~s}$ wit direct solver and $38 \mathrm{~s}$ with the interactive solver.

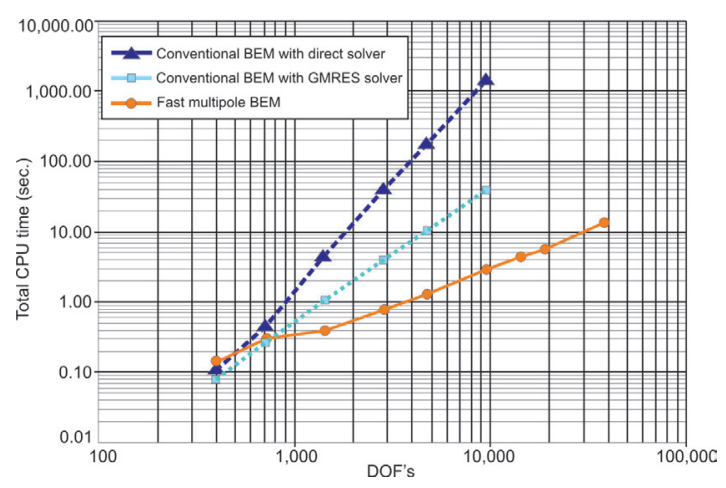

Fig. 8: CPU time used by the conventional BEM and FM BEM.

\section{Conclusion}

A new FM BEM formulation for solving large-scale 2-D elastostatic problems is presented in this paper based on the direct BIE and representations of the kernels with complex analytic functions. The new formulation is shown to be linked directly to the FM BEM for 2-D potential problems. As in the 2-D potential case, complex potentials in the 2-D. elasticity are employed to represent the integrations of the kernels in the in the far field yielding a very compact formulation.

The resulting moment-to-moment, moment-tolocal translations are identical to those for the 2-D potential problems and are symmetrical about the two sets of moments. Thus, programming is straightforward for the new 2-D elasticity FM BEM based on any fast MP BEM code for 2-D potential problems. Two numerical examples are presented that clearly demonstrate the accuracy and efficiency of the developed FM BEM for solving 2-D elasticity problems. From this, it is clear that FM BEM is suitable for modelling many large-scale problems.

Table 1: Computed hoop stress $\sigma_{\theta}$ on the edge of the hole.

\begin{tabular}{|l|l|l|l|l|l|}
\hline \multicolumn{3}{l}{ Fast multipole BEM } & \multicolumn{3}{l|}{ FEM } \\
DoFs & At point A & At point B & DoFs & At point A & At point B \\
\hline 560 & 3.215 & -1.176 & 1206 & 3.148 & -1.101 \\
\hline 920 & 3.216 & -1.183 & 4522 & 3.229 & -1.185 \\
\hline 1640 & 3.216 & -1.185 & 9490 & 3.225 & -1.187 \\
\hline 3080 & 3.217 & -1.188 & 38440 & 3.226 & -1.192 \\
\hline 7600 & 3.222 & -1.190 & & & \\
\hline
\end{tabular}

\section{Acknowledge}

The authors gratefully acknowledge the support by the Slovak Science and Technology Assistance Agency registered under number APV-0169-07, the Slovak Grant Agency VEGA 1/0657/09.

\section{References}

[1] Trebuňa, F., Šimčák, F., Bocko, J., Pástor, M., Stress analysis of casting padestal supporting Structure, Acta Mechanica Slovaca, vol. 13, No.1, p. 36-43.

[2] Horyl, P., Kozoubek, T., Markopoulos, A., Brzobohatý, T., Demonstration of the Matsol library development for the efficient solution of contact problems and its comparison with ANSYS, Acta Mechanica Slovaca, Vol. 14, No.1, 2010, p. 36-42.

[3] Sládek J., Sládek V., Jakubovičová L., Application of Boundary Element Methods in Fracture Mechanics, University of Žilina, Faculty of Mechanical Engineering, Žilina, 2002.

[4] Greengard L. F., Kropinski M.C., Mayo A. , Integral equation methods for Stokes flow and isotropic elasticity in the plane, Journal of Computational Physics 1996, vol. 125, p. 403-414.

[5] Peirce A., P., Napier, J.A.L., A spectral multipole method for efficient solution of large-scale boundary element models in elastostatics, Int J Numer Meth Engng, 38, pp.4009-4034.

[6] Kompiš, V., Štiavnický, M., Kompiš, M., Žmindák, M.: Trefftz interpolation based multi-domain boundary point method, Engineering Analysis with Boundary Elements 29 (2005), p. 391-396.

[7] Kompiš V., Štiavnický M., Žmindák M.:, Murčínková Z., Trefftz radial basis functions (TRBF), Computer Assisted Mechanics 


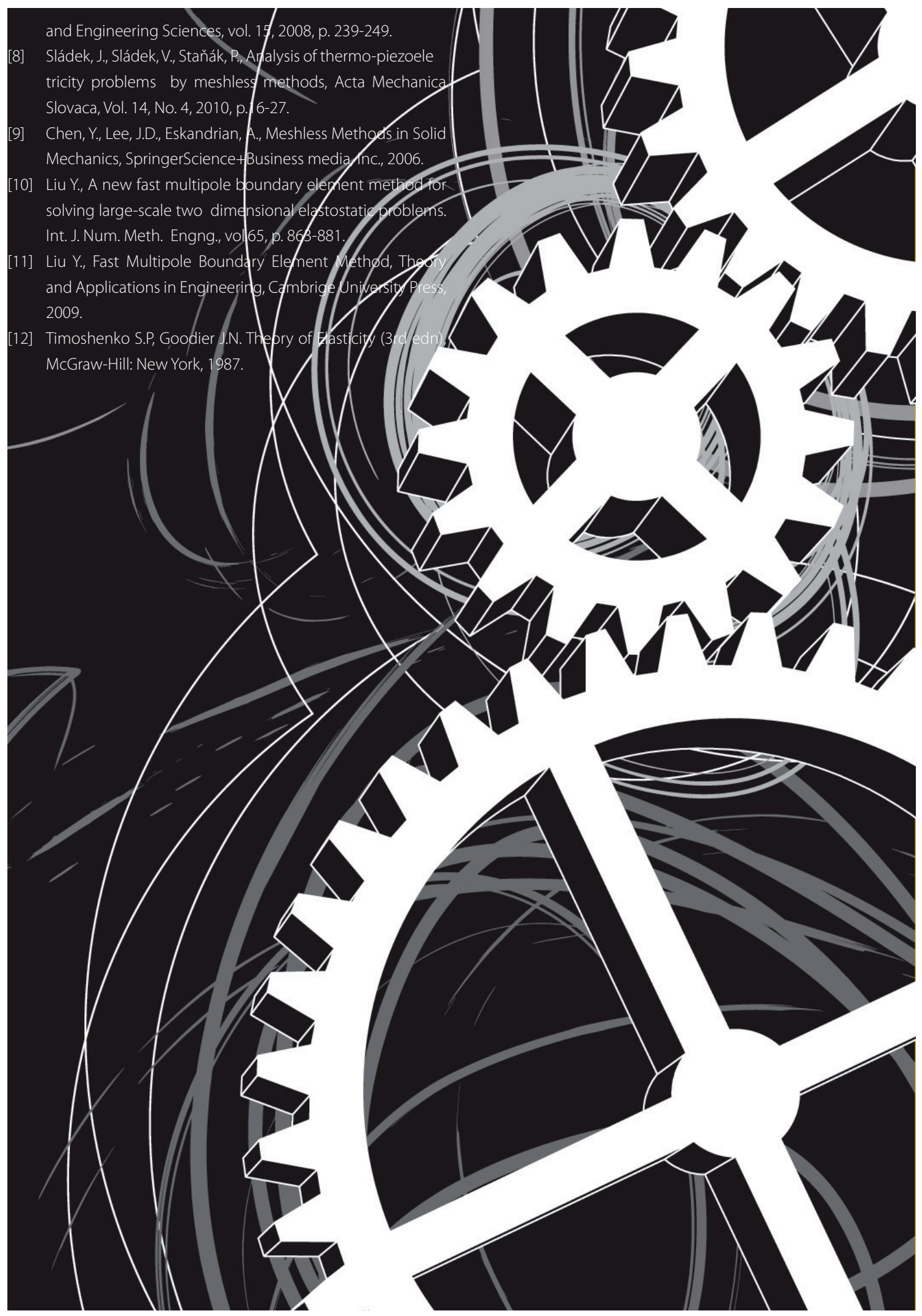

